\title{
The prevalence of hypotension and hypoxaemia in blunt traumatic brain injury in the prehospital setting of Johannesburg, South Africa: A retrospective chart review
}

\author{
W Stassen, BTech (EMC), MPhil EM; T Welzel, MB ChB, DipPEC (SA), HDipIntMed (SA), EMDM (Piedmont), MMedSc (Clin Epi)
}

Division of Emergency Medicine, Faculty of Health Sciences, University of Cape Town, South Africa

Corresponding author: W Stassen (stassen88@gmail.com)

Background. Each year, $89000(180 / 100$ 000) new cases of head injury are reported in South Africa (SA), with the majority of patients being in the economically active population. Hypotension and hypoxaemia significantly increase the morbidity and mortality in patients who have suffered a traumatic brain injury (TBI). Cerebral tissue is particularly vulnerable to these secondary insults in the period immediately following a TBI, emphasising the importance of prehospital care in TBI.

Objective. To establish the prevalence of prehospital hypotension and hypoxaemia in moderate to severe blunt TBI in greater Johannesburg, Gauteng, SA.

Methods. The records of adult patients who sustained a moderate to severe TBI between 1 January and 31 December 2011 were retrospectively reviewed for hypotension (systolic blood pressure $<90 \mathrm{mmHg}$ ) and hypoxaemia (oxygen saturation $<90 \%$ ) during their prehospital phase of care. These results were subject to descriptive analysis.

Results. A total of 299 records were identified, 66 of which met the inclusion criteria. The prevalence of prehospital hypotension and hypoxaemia were $33.3 \%(n=22)$ and $37.9 \%(n=25)$, respectively, while $21.2 \%(n=14)$ of patients suffered double insults of hypotension and hypoxaemia. Hypotension and hypoxaemia were associated with haemorrhage $(p=0.011)$ and chest injuries $(p=0.001)$, respectively.

Conclusion. The prevalence of hypotension in this study was similar to that observed in international studies, but the prevalence of hypoxaemia was much higher. There is a need for local guidelines to be developed to inform the quality of TBI care in the context of the developing world.

S Afr Med J 2014;104(6):424-427. DOI:10.7196/SAMJ.7494

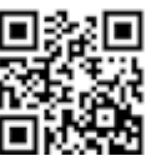

Traumatic brain injury (TBI) describes any injury of the intracranial structures and cerebral parenchyma that might result from trauma to the head. Included in this definition is the cascade of pathophysiological events that lead to progressive worsening of the initial injury such as intracranial haemorrhage and cerebral oedema. ${ }^{[1]}$ TBI may result in altered brain function and present with confusion, altered consciousness, coma, convulsions and/or focal neurology ${ }^{[1]}$

According to the National Institute for Occupational Health, ${ }^{[2]}$ each year $29000(180 / 100000)$ new cases of head injury (of any severity) are reported in South Africa (SA). Of these cases, $50 \%$ were due to road traffic collisions (bicycle, vehicle or pedestrian), $25 \%$ to falls and a further $20 \%$ to violence. ${ }^{[2]}$

Hypotension and hypoxaemia have been found to significantly increase mortality in patients who have suffered a TBI. ${ }^{[1,3 \cdot 7]}$ In one of the largest US-based studies on the effect of hypotension and hypoxaemia on mortality in TBI, the prevalence of hypotension was $34.6 \%$ in the period from injury to the end of emergency centre resuscitation. ${ }^{[4]}$ Hypotension was associated with a doubling in mortality from $27 \%$ in normotensive patients to $64.8 \%$ in their hypotensive counterparts; conversely, favourable outcomes decreased from $51.5 \%$ to $19.4 \%{ }^{[4]}$ Another study reported an eight-fold increase in the relative risk of mortality when a patient had $\geq 2$ hypotensive episodes during his/her recovery ${ }^{[5]}$ Jeremitsky et al. ${ }^{[6]}$ found that patients who died had $50 \%$ more hypotensive episodes than survivors, that hypotensive patients had a longer length of hospital stay, and that fewer hypotensive patients made it to discharge. Hypotension was also independently associated with mortality after multivariate analysis. ${ }^{[0,7]}$

The effects of hypotension on outcome are particularly pronounced in the acute stages following $\mathrm{TBI}^{[8]}$ A more recent study - the International Mission for Prognosis and Analysis of Clinical Trials in TBI (IMPACT) study - combined the results of numerous randomised controlled trials (RCTs) conducted among moderate and severe TBIs in a meta-analysis to establish the relationship between hypoxia and hypotension at admission or in the prehospital phase and outcome. ${ }^{[8]}$ Seven studies were included in the meta-analysis and yielded a combined sample size of 6629 patients. The IMPACT study used proportional odds modelling to show that patients who had hypotensive episodes before or at admission had worse outcomes (odds ratio (OR) 2.7). ${ }^{\left[{ }^{[s]}\right.}$

Most TBIs are followed by an epsiode of apnoea, even if just for a brief period. ${ }^{[10]}$ Numerous studies have revealed the impact that hypoxaemia (partial pressure of oxygen $\left(\mathrm{PaO}_{2}\right)<60 \mathrm{mmHg}$ or oxygen saturation $\left.\left(\mathrm{SpO}_{2}\right)<90 \%\right)$ has on the eventual outcome of patients who have sustained a TBI. Chesnut et al ${ }^{[4]}$ in their large prospective study found the admission prevalence of hypoxaemia in moderate to severe TBI to be $22.4 \%$. Hypoxaemia was associated with a near doubling in mortality from $27 \%$ to $50 \%{ }^{[4]}$ The association between hypotension, hypoxaemia and their duration and mortality was established using multivariate logistic regression. Nearly a quarter (24.6\%) of patients suffered hypoxic episodes during transport. Hypoxaemia was associated with a higher incidence of mortality (OR 
2.66 ), the mortality rate rising to $37 \%$ v. $20 \%$ among patients without hypoxaemia. Prehospital intubation appeared to have no significant variance on the incidence of hypoxaemia. ${ }^{[9]}$ The association between hypoxaemia and mortality in TBI is not as strong as that between hypotension and mortality, but should the two occur simultaneously, the mortality might be as high as $75 \%$. $^{[6]}$

Cerebral tissue is particularly vulnerable to these secondary insults in the period immediately following a TBI. ${ }^{[10-11]}$ A case can be made for earlier intervention to avoid these insults, indicating the importance of prehospital care in TBI. These findings were corroborated in an Australian study that described an additive dose relationship between hypotension and mortality, i.e. the longer the patient remained hypotensive, the worse the outcome. ${ }^{[6]}$ The effects of hypotension on outcome are particularly pronounced in the acute stages following TBI. ${ }^{[8]}$

This study was primarily aimed at establishing the prevalence of prehospital hypotension and hypoxaemia in patients with moderate to severe blunt TBIs in greater Johannesburg, Gauteng, SA.

\section{Methods}

A retrospective, cross-sectional descriptive study was conducted by three methods: $(i)$ an electronic search through a billing database for International Classification of Diseases 10th revision codes that match the description for 'injuries to the head' (S00-S09); (ii) an electronic database search through university student patient report forms for any motor vehicle collision, pedestrian-vehicle collision, fall, assault, explosion or sport-related injury; and (iii) a manual search through helicopter patient report forms. Ethical clearance was granted by the Human Research Ethics Committee, Faculty of Health Sciences, University of Cape Town.

All patient records were limited to adult patients ( $\geq 18$ years) who were seen between 1 January and 31 December 2011 and had a Glasgow Coma Score (GCS) of $\leq 13 / 15$. All transfers and paediatric patients were excluded from the search. If any essential pieces of data were missing (GCS, blood pressure (BP) or saturation measurements) or if the patient died before emergency medical services (EMS) arrival, the patient record was excluded. Cases were further scrutinised for clinical evidence of blunt TBI and any cases not fitting this criterion were excluded (Fig. 1). A blunt mechanism of trauma was selected, as this is the predominant presentation that paramedics face. The highest immediate gain might therefore be achieved by improving the management practices for this more common presentation over those for any penetrating mechanism of trauma.

Demographic characteristics such as age, gender, race, mechanism of injury and concomitant injuries were extracted from each record. The presence of hypoxaemia (defined as $\mathrm{SpO}_{2} \leq 90 \%$ ) and/or hypotension (defined as a systolic blood pressure (SBP) $\leq 90 \mathrm{mmHg}$ ) was captured for each record. The initial $\mathrm{SpO}_{2}$ and $\mathrm{BP}$ readings were recorded (initial prevalence), including the lowest reading for each (prehospital prevalence). Finally, ancillary data regarding the prehospital treatment that these patients received were recorded. Data were subject to descriptive analysis and the association between hypotension or hypoxaemia and injuries sustained was examined using a $\chi^{2}$ test.

\section{Results}

A total of 299 patient records were identified from the initial data search and 66 records matched the inclusion and exclusion criteria (Fig. 1). All records $(n=14)$ included from the second data collection method were void of demographic data and data regarding concomitant injuries and treatment. Table 1 describes the demographics and other data of the sample. The median

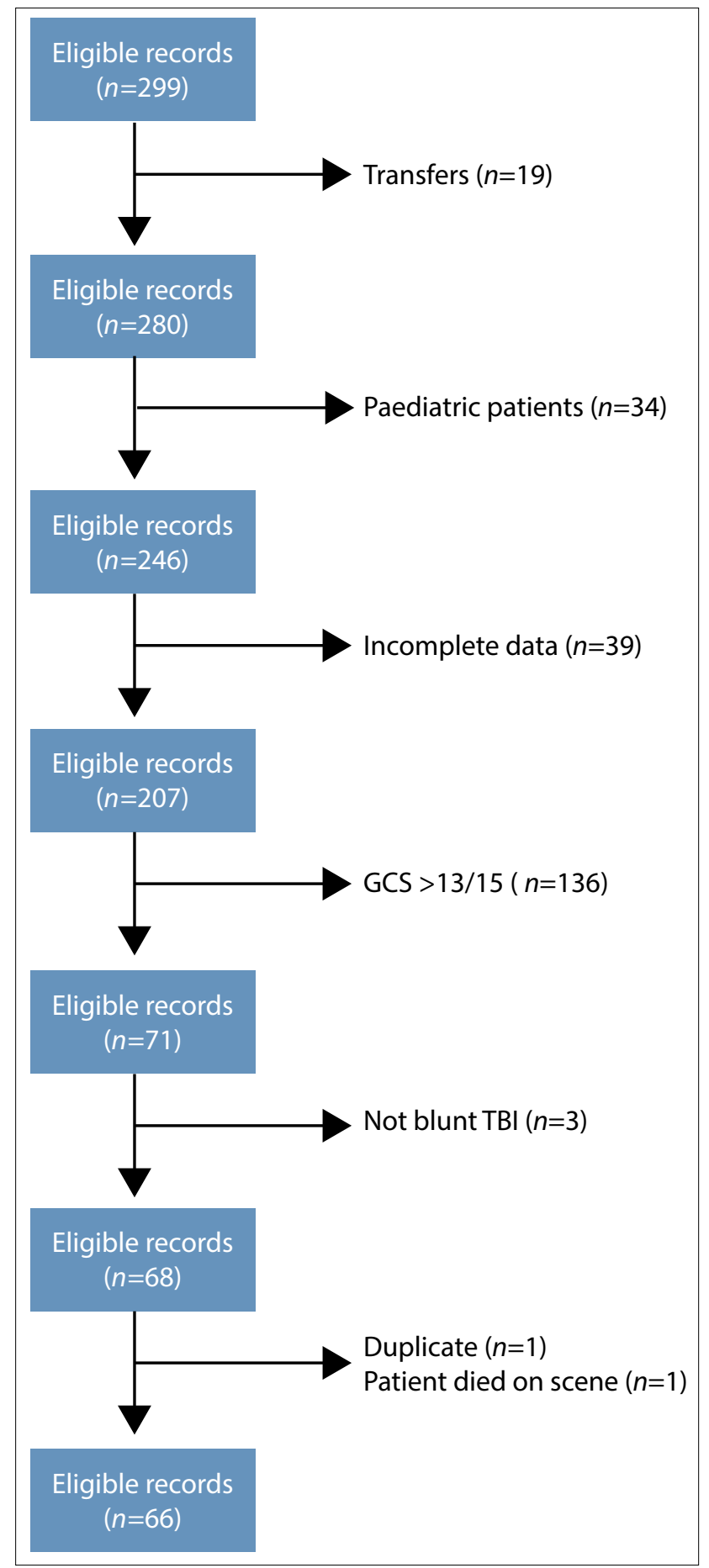

Fig. 1. Study flowchart. $(G C S=$ Glasgow Coma Score; $T B I=$ traumatic brain injury.)

GCS was 5/15. The prevalence of hypoxaemia and hypotension is summarised in Fig. 2. The mean ( \pm standard deviation (SD)) initial SBP was $114.2 \mathrm{mmHg}( \pm 38.4)$ and the mean $( \pm \mathrm{SD})$ lowest SBP was $106.7 \mathrm{mmHg}( \pm 34.8)$. Initial and prehospital hypoxaemia occurred in $37.9 \%(n=25)$ of patients. Finally, the mean $( \pm \mathrm{SD}) \mathrm{SpO}_{2}$ was $90.2 \%$ $( \pm 18.6)$ and $89.65 \%( \pm 18.42)$ initially and at its lowest, respectively.

There was a strong association between injuries and hypotension and hypoxaemia. Of patients with hypotension, $86.7 \%(p=0.011)$ had injuries that had the potential to cause hypotension and of those 
with hypoxaemia, $42.1 \%(p=0.001)$ had a chest injury that could be a likely cause of hypoxaemia.

Table 1. Sample description $(N=66)$

\begin{tabular}{ll}
\hline & $\boldsymbol{n}(\%)$ \\
\hline Gender & \\
Male & $43(66)$ \\
Female & $8(12)$ \\
Unspecified & $15(22)$ \\
Race & \\
Black & $33(51)$ \\
White & $14(21)$ \\
Indian & $1(2)$ \\
Unspecified & $18(27)$ \\
Mechanism of injury & \\
Road traffic collision & $28(42)$ \\
Pedestrian accident & $9(14)$ \\
Motorbike accident & $5(8)$ \\
Assault & $4(6)$ \\
Bicycle accident & $4(6)$ \\
Fall & $1(2)$ \\
Falling object & $1(2)$ \\
Unspecified & $14(21)$ \\
Mode of transport & \\
Air (helicopter) & $45(68)$ \\
Road (ambulance) & $7(11)$ \\
Unspecified & $14(21)$ \\
Concomitant injury & \\
Chest injury & $11(16)$ \\
External haemorrhage & $18(27)$ \\
Facial fracture & $8(12)$ \\
Major fracture & $19(28)$ \\
Occult haemorrhage & $8(12)$ \\
Isolated head injury & $10(15)$ \\
Unspecified & $14(21)$ \\
& \\
\hline
\end{tabular}

\section{Discussion Hypotension}

An SBP of $<90 \mathrm{mmHg}$ was seen in $27.3 \%$ $(n=18)$ of patients at initial presentation, while $33.3 \% \quad(n=22)$ had hypotension at some point during their prehospital phase of care. This prevalence is almost identical to that seen by Chesnut et al., ${ }^{[4]}$ who reported a $34.6 \%$ prevalence. This contrasts with a decade-old study from Cape Town that identified a hypotension rate of $8.3 \% .{ }^{[12]}$ The Cape Town-based study included patients who were transferred from referring facilities and many patients who did not have prehospital data available. Episodes of hypotension might have already been corrected in the referring facilities or went unrecorded, potentially leading to an underestimation of hypotension. The prevalence of hypotension is more than double (68\%) in the study by Manley et al., ${ }^{[5]}$ but their study period, however, included the first 24 hours following admission, making comparisons difficult.

A statistically significant proportion (86.7\%; $p=0.011)$ of patients with hypotension had concomitant injuries that had the potential to cause haemorrhage and hypotension. This finding is supported in the literature that suggests hypotension in TBI typically has a haemorrhagic aetiology, and that TBI as a sole cause of hypotension is only seen in $8.5 \%{ }^{[3]}-13 \%{ }^{[13]}$ of patients. It has been suggested that hypotensive patients with TBI should be approached as if they were suffering from haemorrhagic shock. ${ }^{[13]}$

More patients developed hypotension than originally presented with low BPs, raising the question of whether paramedics responded adequately to a decrease in BP. To investigate this, we performed a $\chi^{2}$ test to establish whether a relationship existed between fluid volume and morphine and midazolam dosages administered and hypotension. The mean (range) total fluid volume that the

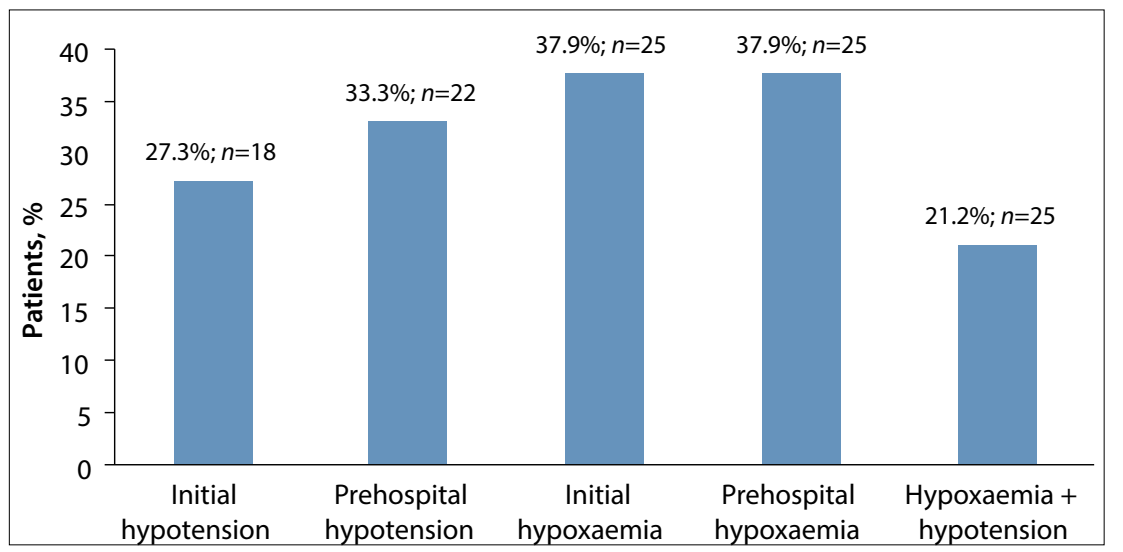

Fig. 2. Prevalence of hypotension and hypoxaemia.

patients received in the prehospital period was $919.2 \mathrm{ml}(0$ - 1 921.1). This fluid therapy had no statistically significant effect on the prevalence of hypotension regardless of the volume infused $(p=0.15)$. As was to be expected, patients who were hypotensive were given more fluid than those who were not, probably in an attempt to correct the hypotension. This difference did not reach statistical significance.

Aggressive fluid resuscitation is recommended in patients with isolated TBI to prevent hypotension, ${ }^{[14]}$ but greater caution is necessary when there are potential sites of uncontrolled haemorrhage. ${ }^{[14]}$ The mean fluid volume of this sample is comparable to that administered to TBI patients in an international study $(1056 \mathrm{ml})$ who had a much lower incidence of hypotension (18\%) at these volumes. ${ }^{[15]}$ If the administered fluid volume was adequate, these patients might have benefited from the use of inotropes to improve their BP and cerebral perfusion pressure (CPP). However, the benefit of inotropes may be offset by the risk of hypertension and the potential for developing acute respiratory distress syndrome. ${ }^{[16]}$

The mean $( \pm \mathrm{SD})$ morphine dose was $8.1 \mathrm{mg}( \pm 5.5)$. The current data showed no association between the morphine dose administered and the prevalence of hypotension $(p=0.94)$. However, a systematic review of RCTs in 2011 found that bolus doses of morphine might be deleterious to outcome by increasing intracranial pressure and decreasing CPP. ${ }^{[17]}$ The mean $( \pm \mathrm{SD})$ midazolam dose $(9.7 \mathrm{mg}( \pm 7.2))$ was higher than that of morphine. A statistically significant association between the midazolam dose and the prevalence of hypotension ( $p=0.009$ ) was found, in keeping with international studies. ${ }^{[18]}$ Unfortunately, it was not possible to compare drug doses or infused volumes against body weight, as recorded data was too unreliable.

\section{Hypoxaemia}

The mean $( \pm \mathrm{SD})$ lowest $\mathrm{SpO}_{2}$ was $89.65 \%$ $( \pm 18.42)$. The prevalence of initial and prehospital hypoxaemia was $37.9 \%$ $(n=25)$. Interestingly, the prevalence in this Johannesburg-based sample was much higher than that reported in the literature $(22.4 \%),{ }^{[4]}$ even though $81 \%$ of patients were intubated. Of patients with hypoxaemia, $42.1 \%$ had a concomitant chest injury that could be responsible for hypoxaemia. As with hypotension, this relationship was statistically significant $(p=0.001)$.

Despite the controversies in prehospital intubation, the US Brain Trauma Foundation 
recommends that all patients with a GCS of $\leq 9 / 15$ who do not have a self-maintained airway, or where pulse oximetry readings remain $\leq 90 \%$, receive aggressive airway management by the most appropriate practicable means taking into account the skill and level of experience of the prehospital care provider and the milieu of the trauma system in which she/he functions. ${ }^{[1]]}$ Locally, there are no published protocols that guide prehospital intubation in the TBI patient and research in this area is lacking. An observational study from the Western Cape did, however, identify an increased mortality in TBI patients who underwent prehospital intubation. ${ }^{[19]}$ Other factors that have been associated with an increase in mortality (injury severity, BP, hypoand hypercapnia, and hypoxaemia) $)^{[1,3-12]}$ in this population were not reported on. There was no statistical difference in the prevalence of hypoxaemia in patients who were intubated in the field $\mathrm{v}$. those who were not $(p=0.15)$.

\section{Study limitations}

Paramedics may inherently be less willing to record grossly abnormal values and this might lead to reporting bias, underestimating the prevalence of hypotension and hypoxaemia. Often, patients (especially those airlifted) were sedated and intubated on scene and the pre-sedation GCS and pre-intubation saturation levels were not recorded. This falsely lowers the GCS and increases $\mathrm{SpO}_{2}$ levels, which could potentially lead to overestimation of TBI severity and underestimation of the prevalence of hypoxaemia. We retrospectively reviewed charts of cases from 2011, so the data presented might not be entirely contemporaneous. The study was also affected by the inherent limitations of a retrospective design based on data of single local EMS providers, thereby limiting extrapolation to other systems. The relatively small sample size may also affect the ability of this study to reach statistical significance in some measures.

\section{Conclusion}

The prevalence of hypotension and hypoxaemia in the greater Johannesburg area is comparable with that observed in international studies. More patients developed hypotension during their prehospital phase of care than had hypotension at initial presentation. Hypotension was associated with midazolam dosage and the presence of injuries that could result in significant haemorrhage. Similarly, hypoxaemia was associated with injuries to the chest and not with field intubation. The current study pointed towards a critical need for educational interventions and the development of a national TBI protocol for prehospital care providers that is based on sound clinical evidence and can guide decision-making on the most appropriate interventions in the prehospital setting to optimise outcome.

Funding. The research was self-funded by WS.

\section{References}

1. Heegaard W, Biros M. Traumatic brain injury. Emerg Med Clin North Am 2007;25(3):655-678. [http:// dx.doi.org/10.1016/..emc.2007.07.001

2. National Health Laboratory Service. World Head Injury Awareness Day 2011. http://www.nioh.ac.za/? page $=$ topical\&id=13\&rid=56 (accessed 9 March 2014).

3. Chesnut RM, Gautille T, Blunt BA, et al. Neurogenic hypotension in patients with severe head injuries J Trauma 1998;44(6):958-963. [http://dx.doi.org/10.1097/00005373-199806000-00003]

4. Chesnut RM, Marshall LF, Klauber MR, et al. The role of secondary brain injury in determining outcome from severe head injury. J Trauma 1993;34(2):216-222. [http://dx.doi.org/10.1097/00005373199302000-00006]

5. Manley G, Knudson MM, Morabito D. Hypotension, hypoxia, and head injury: Frequency, duration and consequences. Arch Surg 2001;136(10):1118-1123. [http://dx.doi.org/10.1001/archsurg.136.10.1118]

6. Jeremitsky $E$, Omert $L$, Dunham $M$, et al. Harbingers of poor outcome the day after severe brain injury: Hypother hy injury: Hypothermia, hypoxia and hypoperfusion. J Trauma 2003:54(2):312-319. [http://dx.doi. org/10.1097/01.1A.0000037876.37236.D

Moppett IK. Traumatic brain injury: Assessment, resuscitation and early management. Br J Anaesth 1093/bja/aem128]

8. McHugh GS, Engel DC, Butcher I, et al. Prognostic value of secondary insults in traumatic brain injury: Results from the IMPACT study. J Neurotrauma 2007;24(2):287-293. [http://dx.doi.org/10.1089/ neu.2006.0031]

9. Chi JH, Knudson M, Vassar MJ, et al. Prehospital hypoxia affects outcome in patients with traumatic brain injury: A prospective multicentre study. J Trauma 2006;61(5):134-141. [http://dx.doi. org/10.1097/01.ta.0000196644.64653.d8]

0. Stiver SI, Manley GT. Prehospital management of traumatic brain injury. Neurosurg Focus 2008;25(4):1-11. [http://dx.doi.org/10.3171/FOC.2008.25.10.E5]

1. Minardi J, Crocco TJ. Management of traumatic brain injury: First link in the chain of survival. Mt Sinai J Med 2009;76(2):138-144. [http://dx.doi.org/10.1002/msj.20105]

12. Reed AR, Welsh DG. Secondary injury in traumatic brain injury patients - a prospective study. S Afr Med J 2002;92(3):221-224.

13. Mahoney E, Biffl W, Harrington D, et al. Isolated brain injury as a cause of hypotension in the blunt trauma patient. J Trauma 2003;55(6):1065-1069. [http://dx.doi.org/10.1097/01. TA.0000100381.89107.93]

14. Roppolo LP, Wigginton JG, Pepe PE. Intravenous fluid resuscitation for the trauma patient. Curr Opin Crit Care 2010;16(4):283-238. [http://dx.doi.org/10.1097/MCC.0b013e32833bf774]

15. Berlot G, La Fata C, Bacer B, et al. Influence of prehospital treatment on the outcome of patients with severe blunt traumatic brain injury: A single-centre study. Eur J Emerg Med 2009;16(6):312-317. [http://dx.doi.org/10.1097/MEJ.0b013e32832d3aal]

16. Contant $\mathrm{CF}$, Valadka AB, Gopinath SP, Hannay HJ, Robertson CS. Adult respiratory distress syndrome A complication of induced hypertension after severe head injury. J Neurosurg 2001;95(4):560-568 [http://dx.doi.org/10.3171/jns.2001.95.4.0560]

17. Roberts DJ, Hall RI, Kramer AH, et al. Sedation for critically ill adults with severe traumatic brain injury: A systematic review of randomized controlled trials. Crit Care Med 2011;39(12):2743-2751. [http://dx.doi.org/10.1097/CCM.0b013e318228236f]

18. Choi YF, Wong TW, Lau CC. Midazolam is more likely to cause hypotension than etomidate in emergency department rapid sequence intubation. Emerg Med J 2004;21(6):700-702. [http://dx.doi. org/10.1136/emi.2002.004143]

19. Sobuwa S, Hartzenberg HB, Geduld H, Uys C. Outcomes following prehospital airway management in severe traumatic brain injury. S Afr Med J 2013;103(9):644-646. [http://dx.doi.org/10.7196/ SAMJ.7035]

Accepted 18 November 2013. 